\title{
The Influence of Edge Energy on Step Flow Instability for Crystals with Bravais Versus Non-Bravais Lattice Structures
}

\author{
Tim Krumwiede ${ }^{1}$, Khoa D. Dinh ${ }^{2}$, Christian Ratsch ${ }^{3}$ and \\ Tim P. Schulze ${ }^{2, *}$ \\ ${ }^{1}$ Department of Mathematics, Bringham Young University, UT 84602, USA. \\ ${ }^{2}$ Department of Mathematics, University of Tennessee, Tennessee 37996, USA. \\ ${ }^{3}$ Institute for Pure and Applied Mathematics and Department of Mathematics, \\ University of California, Los Angeles, CA 90095-1555, USA.
}

Received 1 December 2018; Accepted 9 January 2019

\begin{abstract}
We examine the meandering instability for prismatic lattices formed from triangles, squares and hexagons using a nearest neighbor kinetic Monte Carlo model. In the first two cases, which are Bravais lattices, we find that facets with the orientation favored in the equilibrium shape of isolated islands are most prone to this instability, while the analogous facet for the hexagonal lattice is the least unstable. We argue that this is due to a significant difference in the reconstructed/equilibrium versus the nonreconstructed edge energy for non-Bravais crystals. Surface/edge energy is typically modeled as a single-valued function of orientation. We put forward a simple geometric argument that suggests this picture is inadequate for crystals with a non-Bravais lattice structure. In the case of a hexagonally structured lattice, our arguments indicate that the non-reconstructed edge energy can be viewed as both discontinuous and multi-valued for a subset of orientations that are commensurate with the crystal structure. We support these conclusions with density functional theory calculations that also reveal multivalued surface energies for the set of singular orientations.
\end{abstract}

AMS subject classifications: 82D25

Key words: KMC, surface energy, epitaxy.

\section{Introduction}

In this paper we use kinetic Monte Carlo (KMC) simulations and their underlying lattice models to explore the way surface energy behaves for Bravais versus non-Bravais

*Corresponding author. Email addresses: timwiede@gmail.com (T. Krumwiede), kdinh@vols.utk.edu (K. D. Dinh), cratsch@ipam.ucla.edu (C. Ratsch), tschulze@utk.edu (T. P. Schulze) 
crystals. The effect we identify would appear to be generic to non-Bravais systems, but we illustrate it in detail for a nearest-neighbor model with hexagonally structured layers. Our reason for examining this particular case is that it is the hexagonal analog of the ubiquitous solid-on-solid or, more descriptively, cube-on-cube model that has been used to understand many fundamental aspects of epitaxial growth.

In continuum models the term surface energy normally refers to the surface free en$\operatorname{ergy} \gamma_{f}$, so that it includes entropic effects within some thermodynamic ensemble. This is modeled as a continuous function of surface/edge orientation, $\gamma_{f}(\hat{\mathbf{n}})[1,2]$, and this function is often constructed so that it is consistent with underlying symmetry constraints combined with experimental observations or data from computations $[3,4]$. In contrast, the surface energies $\gamma$ derived from bond counting models are zero-temperature surface energies - entropic effects are introduced via the rates in the KMC model. Most surface energies computed with density functional theory (DFT) are also zero-temperature results.

Often there is a further distinction between equilibrium/reconstructed and nonreconstructed surface energies of ideally truncated planar surfaces. In a lattice based bond-counting model, one can define an unreconstructed surface energy $\bar{\gamma}(\mathbf{n})$ as the number density of broken bonds formed by cutting along a perfectly flat plane or line. In a DFT calculation, one can define a similar quantity where, after the cut is made, the surface is allowed to relax by strict energy descent to a local minimum. This is often a meta-stable configuration. Alternatively, one can define a reconstructed surface energy $\tilde{\gamma}(\mathbf{n})$ by allowing the atoms to reconfigure after the cleaving surface/edge is introduced, so that a global minimum is obtained. For the bond-counting models, one would do this subject to the lattice constraint, whereas the DFT calculations would be constrained by periodic boundary conditions. While the non-reconstructed surface energies defined using metastable states may play no role in the equilibrium behavior of the system, the KMC simulations presented below suggest they are important for the dynamics of some non-equilibrium processes. We find that the non-reconstructed surface energy of nonBravais crystals is both multivalued and discontinuous. This is due to the fact that some orientations give rise to a translation invariant pattern of broken bonds, while others do not.

After deriving the surface energies, as defined above, for the triangular, cubic and hexagonal lattices, we will present a few DFT calculations that support our conclusions before moving on to exploring step-flow dynamics during the epitaxial growth of many layers of each crystal structure. The KMC simulations reveal that something close to the equilibrium/reconstructed behavior is rapidly achieved in many scenarios. For example, if we consider the surface energy of uniformly propagating steps at various crystal orientations, we find near-equilibrium behavior even for large deposition rates. To get at the more subtle behavior of the non-Bravais, hexagonal system, we turn to examining the meandering, or Bales-Zangwill [5], instability, which strongly couples the morphology of the film to the surface energy. This instability occurs in systems with a large stepedge, or Ehrlich-Schwoebel $[6,7]$, barrier that inhibits the interlayer motion of diffusing 
adatoms. As a result, outwardly perturbed portions of a step that have better access to the supply of atoms coming from their lower side tend to grow, or form meanders. This effect is stabilized by a large edge energy, which favors flatter step-edges. As a result, the most unstable orientations are those with the lowest edge energy. For the Bravais lattices, there is no distinction between the equilibrium/reconstructed and the non-reconstructed surface energy, as all orientations have a translation invariant broken bond density and there is no reconstruction of a truncated surface/edge for the nearest neighbor models. In contrast, for the hexagonal case, it is the non-reconstructed energy of a straight step that correctly predicts which orientation is most unstable.

\section{The edge energies}

We start by examining the nearest-neighbor bond-counting models for three lattices: a simple-cubic lattice, and analogous stacked, triangular and hexagonal prisms. The first two of these are Bravais lattices, which have points $\left\{\mathbf{x} \in \mathbb{R}^{d}\right\}$ that are integer combinations of $d$ independent primitive vectors $\left\{\mathbf{e}_{i}\right\}_{i=1}^{d}$, where $d$ is the number of dimensions:

$$
\mathbf{x}=\sum_{i=1}^{d} n_{i} \mathbf{e}_{i} .
$$

For the simple cubic lattice, one can scale lengths so that the primitive vectors are just the Cartesian unit vectors, $\hat{\mathbf{i}}, \hat{\mathbf{j}}$ and $\hat{\mathbf{k}}$.

A non-Bravais lattice, often referred to as a "lattice plus a basis" [8] or an $n$-lattice with $n>1$ [9], can be represented by a fixed arrangement of $n$ lattice points for each point in an underlying Bravais lattice:

$$
\left\{\mathbf{x}+\mathbf{y}_{i}\right\}_{i=1}^{n} \text {. }
$$

The simplest example is the hexagonal lattice, which models the idealized graphene structure. In this case, the underlying Bravais lattice is the triangular lattice. There are two hexagonal lattice points associated with each point in the triangular lattice, which one can take to be $\mathbf{y}_{1}=\mathbf{0}$ and $\mathbf{y}_{2}=\frac{1}{2} \mathbf{i}+\frac{\sqrt{3}}{6} \mathbf{j}$. We will also consider the three dimensional hexagonal prism formed by stacking layers of the hexagonal lattice. This can be loosely thought of as a model of graphite, which is formed by alternating, offset layers of graphene. Effectively, we would be ignoring the interlayer bonds responsible for the offset. These are the result of relatively weak van der Waals forces, as opposed to the much stronger covalent bonds that form the intralayer bonds. Our primary aim, however, is not to model a specific material, but to make the most straightforward generalization of the basic cube-on-cube model.

In a pairwise bond-counting model, an energy is defined for a given lattice configuration by defining sets of bond orientations $\mathrm{V}=\left\{\left\{\mathbf{v}_{i j}\right\}_{j=1}^{J_{i}}\right\}_{i=1}^{N_{p}}$ and corresponding bond energies $e_{i j}$ for each of the $N_{p}$ particles in the system [10]. These sets are often restricted to neighboring pairs of atoms, but, in principle, could include all combinations of atoms. 
For a crystal with a Bravais lattice structure, the same set of bonds, $\left\{\mathbf{v}_{j}\right\}_{j=1}^{J}$, applies to each particle in the crystal. In this case, one can show that all orientations yield a translation invariant surface energy and, for the nearest-neighbor model, there is no reconstruction. The surface/edge energy of bond-counting models on Bravais lattices is given by

$$
\bar{\gamma}(\hat{\mathbf{n}})=\frac{1}{2} \sum_{j=1}^{J} e_{j} \frac{\left|\hat{\mathbf{n}} \cdot \mathbf{v}_{j}\right|}{|\operatorname{det} A|}
$$

where $\hat{\mathbf{n}}$ is the normal to the surface/edge and $A$ is a matrix with the lattice primitive vectors as columns [11].

The idealized graphene structure is one of the simplest examples of a non-Bravais lattice. For a nearest-neighbor hexagonal model, the particles have one of two distinct sets of bonds, $\left\{\left\{\mathbf{v}_{A j}\right\}_{j=1}^{3},\left\{\mathbf{v}_{B j}\right\}_{j=1}^{3}\right\}$. Gan and Srolovitz were the first to address the issue of edge energy for individual graphene flakes [12]. They use DFT calculations for a collection of graphene ribbons at seven different orientations to interpolate an edge energy function, and consider unreconstructed graphene with both non-terminated and hydrogen terminated bonds, as well as a model for reconstructed graphene. Liu et al. [13] revisit the problem and first consider an arbitrarily oriented graphene edge that can be decomposed into a number of "zigzag" and "armchair" components, so that the edge energy can be represented using two energies of these primary configurations along with zigzag and armchair densities that can be computed from simple geometric considerations:

$$
\tilde{\gamma}(\chi)=\frac{4}{\sqrt{3}} \epsilon_{A} \sin (\chi)+2 \epsilon_{Z} \sin (\pi / 6-\chi),
$$

where $\epsilon_{A}$ and $\epsilon_{Z}$ are the energies of an atom in an armchair or zigzag component respectively and $\chi$ is the edge angle. This assumption is equivalent to assuming edges of the graphene flake reconstruct, so that they do not contain singly-bonded carbon-atoms. This same assumption appears to have been tacitly made in [12], as a perfectly linear edge with the slope indicated in their Fig. $2 b$ would have an additional singly-bonded atom at the kinks along the edge. This assumption is appropriate for a system near equilibrium, as the singly bonded atoms will quickly restructure to form double bonds. While it is not surprising that equilibrium shapes are dominated by facets without these dangling atoms, it seems clear they would appear in non equilibrium structures and could affect the dynamics of relaxation and growth processes. Indeed, Artyukhov et al. [14] go on to consider growth mechanisms involving singly-bonded carbon atoms arriving at and diffusing along steps similar to what occurs in the traditional Burton-Cabrera-Frank [15] theory of step-flow on surfaces, and singly-bonded atoms at graphene edges have been observed in experiments [19]. In view of this, we examine a more complete picture of surface/edge energy as a function of perfectly planar/linear facets at arbitrary orientations and positions.

Below, we examine these effects for an isolated hexagonal lattice using nearestneighbor, bond-counting arguments, neglecting reconstructions and other off-lattice effects. The idealized model allows us to gain a level of mathematical clarity, but we expect 


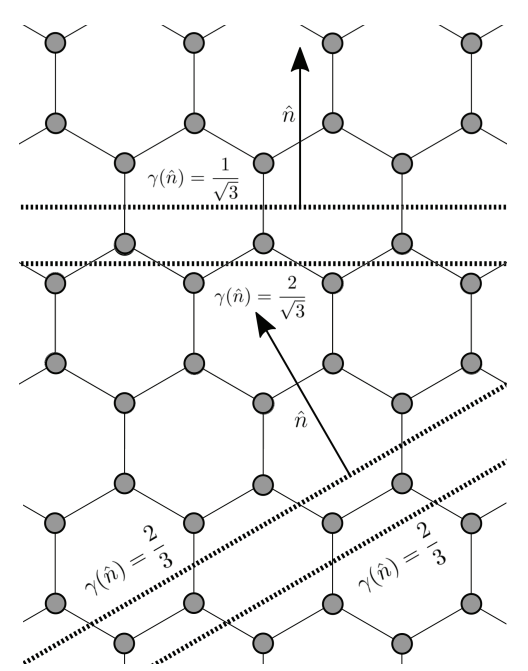

Figure 1: A hexagonal/graphene lattice cut by two lines in the zigzag orientation (near the top of the figure) and two more in the armchair orientation (near the bottom of the figure). In the case of the zigzag orientations, the broken bond density can be altered by a parallel translation of the edge, while the broken bond density is translation invariant for the armchair orientation.

similar conclusions to apply to real materials and first-principles calculations, including graphene films grown on substrates. To make this point clear we have also included a few results of DFT calculations.

For the graphene structure, we will see that including singly bonded atoms leads to a discontinuous, multi-valued edge energy. These singularities occur for a discrete set of orientations that are commensurate with the crystal structure. In the case of graphene, this includes the so-called zigzag orientation, which dominates the equilibrium shape of isolated crystals in the nearest neighbor model.

The mechanism that is responsible for the discontinuities in the surface energy is illustrated in Fig. 1 using a nearest-neighbor bonded crystal with the hexagonal/graphene structure. Most facets behave like the armchair orientation shown at the bottom of Fig. 1, where the broken bond density, which represents the nonreconstructed edge energy in this simple model, is translation invariant. This contrasts with a countable, discrete set of orientations that behave like the zigzag orientation shown at the top of Fig. 1, where the broken bond density alternates between two values as the line cutting the crystal is translated in the normal direction.

In general, edge orientations fall into one of two categories: commensurate orientations result in a periodic pattern of broken bonds, while non-commensurate orientations result in an aperiodic pattern of broken bonds. An edge with a commensurate orientation can be translated so that it passes through multiple sites, while an edge with an incommensurate orientation can pass through at most one site. The commensurate edges give rise to two sub-cases we refer to as congruent and incongruent. While the incommensurate and congruent orientations have translation invariant edge energies, the edge energy for 

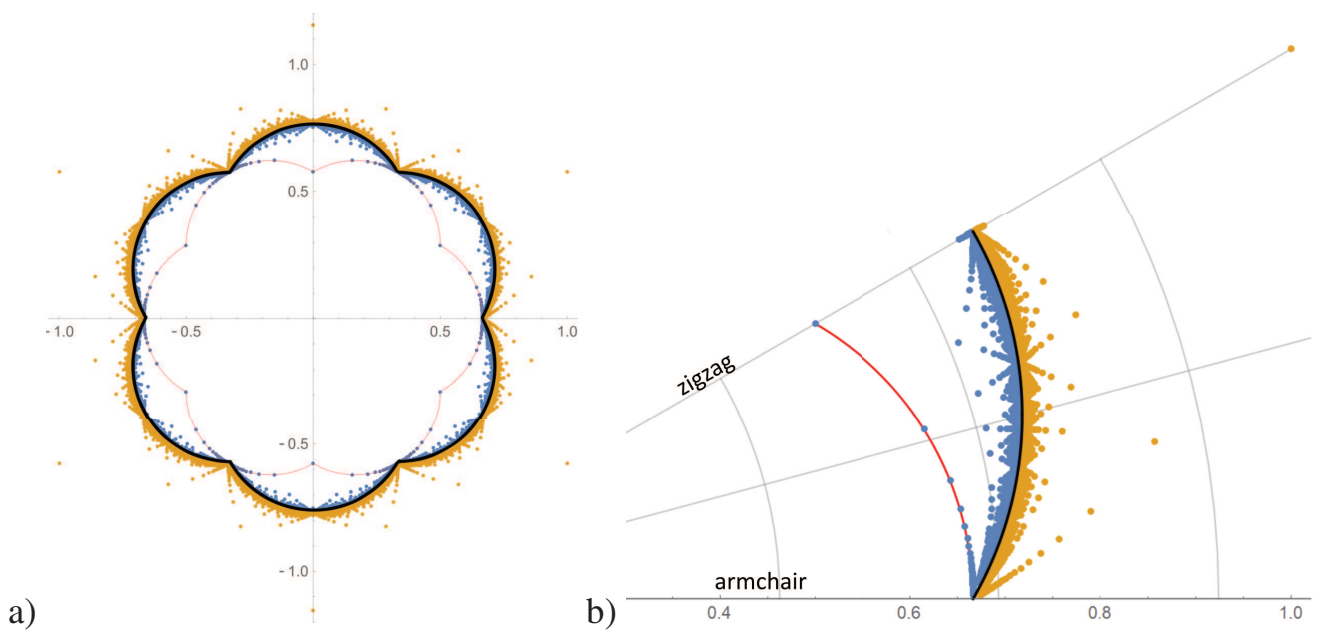

Figure 2: A polar plot of broken-bond density/edge energy for the hexagonal/graphene crystal as a function of edge orientation. The entire plot is shown in (a) while (b) contains only the wedge ranging between armchair and zigzag orientations. Most values lie on the black curve (2.5), while the discrete set of incongruent orientations gives rise to discontinuities with two edge energy values for each orientation: the minimum values are shown in blue and the maximum values are gold. The red curve (2.6) interpolates between the zigzag and armchair orientation by neglecting dangling bonds.

the incongruent orientations is multi-valued. An edge with a congruent orientation can be translated so that it passes through either no sites or sites with both A-oriented and B-oriented bonds, alternating between the two, while an edge with an incongruent orientation can only pass through no sites or sites with the same bond orientations.

These results are summarized in Fig. 2. The black curve, described analytically by

$$
\bar{\gamma}(\chi)=\frac{2}{3 \sqrt{3}} \sum_{n=0}^{2}\left|\cos \left(\chi-\pi / 6+\frac{n}{3} \pi\right)\right|
$$

is the edge energy that applies to the uncountably infinite number of incommensurate and the countably infinite set of congruent edges. This edge energy is exactly $1 / 3$ what one would find for a nearest neighbor model based on the related Bravais lattice with an additional lattice point in the center of each hexagon. This curve is discontinuous at the incongruent orientations, where one finds two possible values of the edge energy depending on the placement of the facet in the normal direction. It can be shown that the average of these two values again lies on the black curve (2.5). Finally, the red curve, given by

$$
\tilde{\gamma}(\chi)=\frac{1}{3} \sum_{n=0}^{2}\left|\cos \left(\chi+\frac{n}{3} \pi\right)\right|
$$

is the edge energy (2.4) derived in Liu et al. [13] with values $\epsilon_{A}=\frac{2}{3}$ and $\epsilon_{Z}=\frac{1}{\sqrt{3}}$ by assuming edges that consist of only armchair and zigzag components. This curve is a 


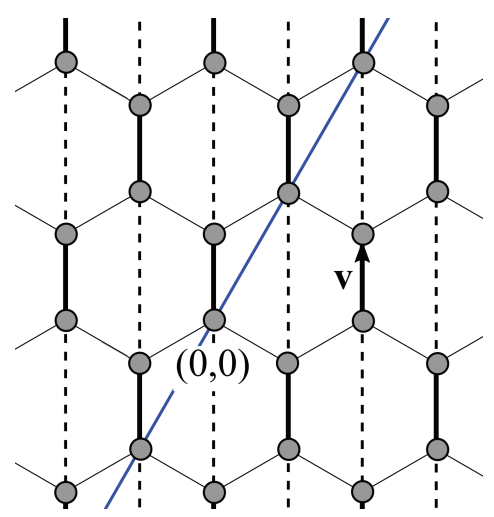

Figure 3: The hexagonal lattice with a single nearest-neighbor bond $\mathbf{v}$ and its corresponding set of bond-lines. The blue reference line $\hat{y}=\sqrt{3} x$ is used to define the sequence $\delta_{n}$ referred to in the text.

lower bound on the defect edge energy, and is formed by continuously interpolating between the lower of the two possible values one can obtain with a zigzag orientation and the single value for the armchair orientation. Note that if this simple, interpolated edge energy function was used to evolve a non-equilibrium shape, one would not expect any qualitative difference in the dynamics compared to that for a material with a triangular lattice structure, i.e. both edge energies are a six-petaled flower.

To get these results, we follow arguments that generalize those of Mackenzie et al. [10], i.e. we compute a contribution to the edge energy for each bond orientation $\mathbf{v}$ and sum the result over all bonds $V$. To this end, consider the set of all bond-lines parallel to $\mathbf{v}$ that pass through lattice sites. Note that the bonds only cover $\frac{1}{3}$ of each bond-line, with a repeating pattern of one bond followed by two bond-less segments (see Fig. 3). Thus, the bonds and bond-line structure are periodic in the vertical direction with period $3 a$, where $a$ is the bond length. We will make use of a Cartesian coordinate system where the $y$-axis is aligned with the bond and the origin is placed at the lower end of an arbitrary bond. It is also convenient to introduce a reference line $\hat{y}=\sqrt{3} x$ and measure distance in the direction of $\mathbf{v}$ relative to this line, $\Delta=y-\hat{y}$.

Next, we consider an arbitrary edge $y=s x+b$ with slope $s$ and intercept $b$. Between any two adjacent bond-lines, this line rises a distance $r=\frac{\sqrt{3}}{2}$ as and intersects the $n^{\text {th }}$ bondline, given by $x=n \frac{\sqrt{3}}{2} a$, at $y_{n}=r n+b$. Relative to the reference line defined above, this produces the sequence $\Delta_{n}=y_{n}-\hat{y}_{n}=\left(r-\frac{3 a}{2}\right) n+b$. We will need to consider $\mathrm{y}$-values mapped to the interval $[0,3 a]$ via congruence modulo $3 a$. It will therefore be convenient to scale distance so that $a=\frac{1}{3}$ and this congruence operation corresponds to taking the fractional part of $y$-values. After scaling, half of the bonds are congruent to the interval $[0,1 / 3]$ while the other half are congruent to the interval $[1 / 2,5 / 6]$. Relative to the reference line, the scaled bond locations will have fractional parts in the interval $[0,1 / 3]$. In order to determine the intersections with bonds, it is sufficient to consider the fractional 
part of the sequence $\delta_{n}=\operatorname{Frac}\left(\Delta_{n}\right)$.

An edge orientation is commensurate with respect to bond $\mathbf{v}$ if $r \in \mathbb{Q}$ and incommensurate otherwise. For a given edge orientation, one can show that all of the bonds $\mathbf{v} \in V$ fall into the same category. For incommensurate orientations, the edge energy is defined as the mean number of bonds cut across the entire edge. A natural hypothesis for this mean is $\gamma(\hat{\mathbf{n}})=\frac{1}{3} \Gamma(\hat{\mathbf{n}})$, where $\Gamma$ is the edge energy for the related triangular lattice with additional nodes in the center of each hexagon, as the graphene lattice is formed by removing $\frac{2}{3}$ of these bonds. The triangular lattice is Bravais, so that $\Gamma$ can be computed from (1).

To see that this is correct, we first consider the case $b=0$, producing the sequence $\delta_{n}=\operatorname{Frac}\left[\left(r-\frac{1}{2}\right) n\right]$. Since $r$ is irrational, so is $r-\frac{1}{2}$ and Weyl's equidistribution theorem [20] then indicates that the sequence is uniformly distributed. This implies that one third of the bond-line intersections correspond to broken bonds. For $b \neq 0, \operatorname{Frac}\left[\left(r-\frac{1}{2}\right) n+b\right]=$ $\operatorname{Frac}\left[\left(r-\frac{3 a}{2}\right) n+\operatorname{Frac}(b)\right]$, from which we can see that broken bond density in the incommensurate case is translation invariant, as the portion of the uniform distribution of $\delta_{n}$ that is shifted out of the interval $[0,1]$ on the right simply reemerges on the left. The same result holds for each $\mathbf{v} \in \mathrm{V}$ and therefore

$$
\gamma(\hat{\mathbf{n}})=\frac{1}{3} \Gamma(\hat{\mathbf{n}})=\frac{2}{3 \sqrt{3}} \sum_{i=1}^{3}\left|\hat{\mathbf{n}} \cdot \mathbf{v}_{i}\right|,
$$

where we have expressed the result using twice the contribution from the three distinct A-bond orientations $\mathbf{v}_{A 1}=(\sqrt{3} / 2,1 / 2), \mathbf{v}_{A 2}=(-\sqrt{3} / 2,1 / 2), \mathbf{v}_{A 3}=(0,1)$, as the B-bonds give rise to the same contributions.

The sequence $\delta_{n}$ is periodic whenever $r$ is rational, repeating every $N$ bond-lines, where $N$ is the smallest even integer such that $N r \in \mathbb{Z}$. When this integer $N$ is divisible by three, we refer to the orientation as congruent, as one can show that congruence applies to all bonds $\mathbf{v} \in V$ or none at all. Congruent orientations have the same translation invariant broken bond density as the incommensurate orientations. To see this, note that the $N$ bond-line intersections are evenly spaced over one period of length $p=r N$ and that the corresponding values of $\delta_{n}$, though re-ordered, are uniformly spaced over the interval $[0,1]$, with one third of these corresponding to a bond crossing.

The remaining commensurate cases have a repeating sequence $\delta_{n}$ with $N \equiv 1$ or 2 mod3. In these cases, which we refer to as incongruent, there is no way to have exactly one third of the $\delta_{n}$ falling into the first third of $(0,1]$. Instead, the number of intersections with bonds per period will round up or down to the nearest integer that is divisible by 3 . If $N \equiv 1 \bmod 3$, the lesser of the two edge energies is given by

$$
\gamma_{\mathbf{v}}^{-}(\hat{\mathbf{n}})=\frac{n-1}{3 p}=\frac{p \Gamma_{\mathbf{v}}(\hat{\mathbf{n}})-1}{3 p}=\frac{1}{3} \Gamma_{\mathbf{v}}(\hat{\mathbf{n}})-\frac{1}{3 p^{\prime}},
$$

and the greater of the two edge energies is

$$
\gamma_{\mathbf{v}}^{+}(\hat{\mathbf{n}})=\frac{1}{3} \Gamma_{\mathbf{v}}(\hat{\mathbf{n}})+\frac{2}{3 p} .
$$


Which of the two applies depends on the intercept $b$ of the dividing line, and the edge energy fails to be translation invariant for these orientations. The transition between the two values takes place whenever the edge crosses a lattice site. If $N \equiv 1 \bmod 3$, this occurs whenever $\operatorname{Frac}(b)=k / N$ or $k / N+1 /(3 N)$ with $k \in \mathbb{Z}$. And if $N \equiv 2 \bmod 3$, when $\operatorname{Frac}(b)=k / N$ or $k / N+2 /(3 N)$. We will refer to the set of edges with $b$ between any two of these transition values as a band. Note that all edges within a single band share the same energy value. There are two possible values for any edge orientation, with bands alternating between the two and one of the bands being twice as wide as the other.

If $N \equiv 2 \bmod 3$, the two edge energy values are

$$
\gamma_{\mathbf{v}}^{-}(\hat{\mathbf{n}})=\frac{1}{3} \Gamma_{\mathbf{v}}(\hat{\mathbf{n}})-\frac{2}{3 p}, \quad \gamma_{\mathbf{v}}^{+}(\hat{\mathbf{n}})=\frac{1}{3} \Gamma_{\mathbf{v}}(\hat{\mathbf{n}})+\frac{1}{3 p} .
$$

The total edge energy for an edge within a thin band is given by

$$
\begin{aligned}
\gamma_{1}(\hat{\mathbf{n}}) & =\sum_{N \equiv 1 \bmod 3} \gamma_{\mathbf{v}_{i}}^{+}(\hat{\mathbf{n}})+\sum_{N \equiv 2 \bmod 3} \gamma_{\mathbf{v}_{i}}^{-}(\hat{\mathbf{n}}) \\
& =\frac{1}{3} \Gamma(\hat{\mathbf{n}})+\frac{2}{3 p}\left(m_{1}-m_{2}\right),
\end{aligned}
$$

where $m_{j}$ is the number of bonds in $\left\{\mathbf{v}_{i}\right\}$ for which $N \equiv j \bmod 3$. Similarly, the edge energy for a edge within a thick band is

$$
\gamma_{2}(\hat{\mathbf{n}})=\frac{1}{3} \Gamma(\hat{\mathbf{n}})+\frac{1}{3 p}\left(m_{2}-m_{1}\right) .
$$

Note that

$$
\lim _{p \rightarrow \infty} \gamma_{1}=\lim _{p \rightarrow \infty} \gamma_{1}=\frac{1}{3} \Gamma,
$$

so that in the limit where the period of the bond intersections becomes large, both of the values for the incongruent orientations converge to the value for incommensurate/congruent orientations.

Let $\gamma^{-}(\hat{\mathbf{n}})=\min \left\{\gamma_{1}(\hat{\mathbf{n}}), \gamma_{2}(\hat{\mathbf{n}})\right\}$ and $\gamma^{+}(\hat{\mathbf{n}})=\max \left\{\gamma_{1}(\hat{\mathbf{n}}), \gamma_{2}(\hat{\mathbf{n}})\right\}$. These two functions are then the minimum and maximum energy values for the orientation $\hat{\mathbf{n}}$ shown in Fig. 2. In particular, the zigzag orientations minimize $\gamma^{-}$over all incongruent orientations. If we perform the classical Wulff construction using $\gamma^{-}$as the edge energy, we get a hexagon with zigzag orientation edges.

\section{Density functional theory results}

We have also done DFT calculations for graphene edges that confirm that multi-valued edge energies for certain orientations are not an artifact of the simple bond-counting model. 
All calculations were performed with the FHI-AIMS package [16]. This is an allelectron full-potential DFT code that uses numeric atom-centered orbitals as its basis set. The exchange-correlation energy was approximated by the GGA-PBE functional [17]. We used light basis set settings as implemented in FHI-AIMS, and included van der Waals interactions as implemented by the Tkatchenko-Scheffler [18] method.

We used a supercell approach where graphene ribbons were bounded by two identical edges on the left and on the right and periodic boundary conditions were enforced at the top and bottom. We carefully tested the width of the graphene ribbons and for all results reported below we used a width of $\sim 15 \AA$. The edge energy was calculated as the difference of a supercell calculation with two edges (as described above) minus the energy of the corresponding number of carbon atoms in a pristine (infinite) graphene sheet. All atoms in the calculations were allowed to relax. We calculated a number of different edge orientations as summarized in Table 1.

Table 1: Edge energy calculations from DFT calculations for a number of edge orientations from armchair to zigzag.

\begin{tabular}{||l|r|r||}
\hline Angle (deg) & Type & Energy \\
\hline $0.0(\mathrm{AC})$ & Congruent & 1.031767446 \\
4.715003954 & Max & 1.109965069 \\
& Min & 1.091758775 \\
6.586775554 & Max & 1.152994339 \\
& Min & 1.116107374 \\
10.89339465 & Max & 1.310235317 \\
& Min & 1.16634251 \\
13.89788625 & Max & 1.227909468 \\
& Min & 1.196419461 \\
16.10211375 & Max & 1.293860808 \\
& Min & 1.222571091 \\
19.10660535 & Congruent & 1.276464491 \\
21.05172444 & Max & 1.293664015 \\
& Min & 1.274587888 \\
22.41091053 & Max & 1.30050333 \\
& Min & 1.285688572 \\
23.41322445 & Congruent & 1.30536139 \\
$30.0(\mathrm{ZZ})$ & Max & 1.923011558 \\
& Min & 1.351281052 \\
\hline
\end{tabular}

Note that in these calculations, the minimum energy occurs for an armchair edge, whereas the nearest neighbor model described in the previous section has the minimum occurring for the lower of the two zigzag edge energies. To get quantitative agreement with the DFT results we would have to include longer range interactions. Nevertheless, we include these results to emphasize the qualitative feature that there are two distinct 
energy values for incongruent edges as the edge is translated. We also note that the DFT results are in good agreement with those of Liu et al. [13].

\section{Kinetic Monte Carlo}

KMC replaces Molecular Dynamics with a Markov Chain, where the states are different configurations for the basins of attraction and the transition rate models are based on the harmonic approximation to transition state theory [21,22]. Ideally, the basins are defined by particle locations in configurations that locally minimize some potential energy function $\Phi\left(\left\{\mathbf{r}_{i}\right\}_{i=1}^{N_{p}}\right)$ for the $N_{p}$-particle configuration space. However, for crystalline materials, these basins adopt an approximate lattice structure, so many models assume an idealized, perfect lattice. The most commonly performed simulations assume a simple cubic lattice, with states represented by occupation arrays on this lattice $s=\left\{s_{i j k} \in\{0,1\}\right\}$. One then associates an energy $E(s)$ with each configuration. For example, the simplest models associate a "bond" energy $e_{b}$ with each pair of occupied nearest neighbors. There is a close analogy between the model just described and the well studied Ising model for a ferromagnet $[23,24]$. Indeed, one can directly map the states and energies from one system to the other. There exists a tremendous variety of generalized models, allowing for longer range interactions, multiple species and many-body interactions.

Transitions are usually restricted to single-atom moves to neighboring unoccupied sites, with rates that depend exponentially on an atom's binding energy:

$$
R=K e^{-n e_{b} / k_{B} T},
$$

where $n$ is the number of occupied nearest neighbor sites, $e_{b}$ is the bond energy just described, $k_{B}$ is Boltzmann's constant, $T$ is the temperature and $K$ is a prefactor that sets the overall timescale of the dynamics. In a solid-on-solid model, one assumes no vacancies or overhangs, so that every surface atom sits on one in the previous layer. In this case, the substrate contribution to the hopping rate can be absorbed into the prefactor $K$. Within this same model, a simple way to incorporate a step edge barrier is to reject a fraction $v$ of interlayer moves. This must be done in both directions to maintain detailed balance. These rates then allow the system to maintain a canonical equilibrium state with the Boltzmann distribution where the probability of being in state $s$ is

$$
P(s)=\frac{1}{Z} e^{-E(s) / k_{B} T},
$$

where $Z=\sum_{s} \exp \left(-E(s) / k_{b} T\right)$ is the canonical partition function, $E(s)$ is the energy associated with state $s, k_{B}$ is Boltzmann's constant, and $T$ is the temperature. These transitions form a continuous-time Markov process governed by the master equation

$$
\frac{d}{d t} P\left(s_{j}\right)=\sum_{i}\left(R\left(s_{i} \rightarrow s_{j}\right) P\left(s_{i}\right)-R\left(s_{j} \rightarrow s_{i}\right) P\left(s_{j}\right)\right),
$$


which has the steady state (4.2) as the result of a detailed balance relationship where the flux between any two states matches, i.e.

$$
R\left(s_{1} \rightarrow s_{2}\right) P\left(s_{1}\right)=R\left(s_{2} \rightarrow s_{1}\right) P\left(s_{2}\right) .
$$

The goal of KMC simulation is to produce a sequence of events that sample the dynamics as quickly as possible. To do this, one must implement the following steps:

\section{KMC Algorithm}

1. Calculate the sum $P_{J}=\sum_{j=1}^{J} R\left(s_{i} \rightarrow s_{j}\right)$, retaining the partial sums $P_{j}$.

2. Choose a random number $r \in\left[0, P_{J}\right)$,

3. Search the list of partial sums until $P_{j-1} \leq r<P_{j}$.

4. Select and execute event $j$.

A fast way of implementing this algorithm is to use an inverted list data structure [25].

\section{$5 \quad \mathrm{KMC}$ results}

Simulations of the meandering or Bales-Zangwill instability using the cube-on-cube model for epitaxial growth are well established [26]. Normally, these simulations assume a stepped surface with steps oriented so that perfectly flat steps are kink-free. This corresponds to interfaces with orientations at a local minimum/cusp in the polar plot of the surface energy as a function of orientation (see Fig. 4). In such simulations, one uses a periodic boundary condition in the direction parallel to the steps and a helical type boundary condition orthogonal to the steps, so that the top and bottom step are smoothly joined. This enforces a topological constraint where the net number of steps down per period is fixed. To consider a broader class of miscut surfaces, we modify the periodic boundary condition so that the net number of oriented kinks along each step can be constrained in a similar way. As one tilts the mean orientation of the steps away from the equilibrium angle, introducing kinks along the step, one raises the surface energy. Perturbations to such interfaces are therefore higher energy and more difficult to maintain, with the result that the tilted interfaces are less prone to the meandering instability.

We illustrate these basic results in Fig. 5 with two surface images - an initial one and one after 80 monolayers (ML) of growth - for each of three tilt angles. The first pair is for growth at the equilibrium orientation and is subject to the greatest degree of instability, while the third one is maximally tilted away from the preferred angle for equilibrium structures and is almost completely stable. These results are further reflected in Fig. 6 where we plot the relative defect energy (broken bond density),

$$
\bar{\gamma}-\bar{\gamma}_{0}=\frac{\sum_{i=1}^{Z-1}(Z-i) N_{i}}{(\text { ideal facet length })(\text { number of steps })}-\bar{\gamma}_{0}
$$




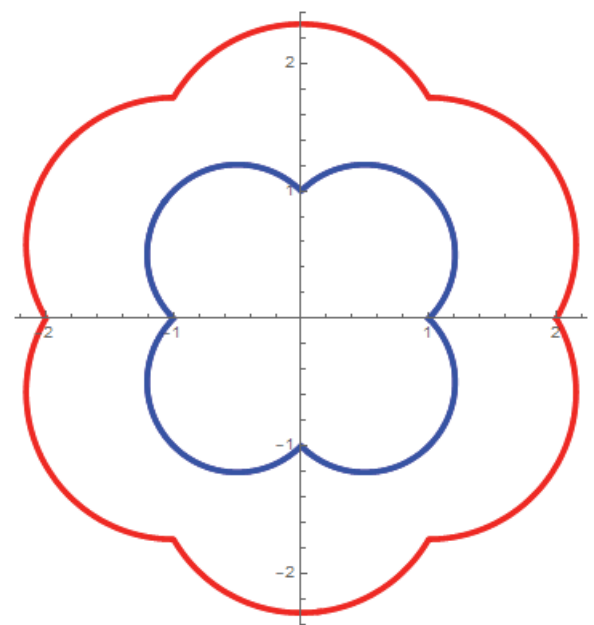

Figure 4: Polar plots of the edge energy functions counting nearest-neighbor bonds for the simple square lattice (in blue) and the triangular lattice (in red). Since both lattices are Bravais, edge energy values are translation invariant, leading to single valued functions.
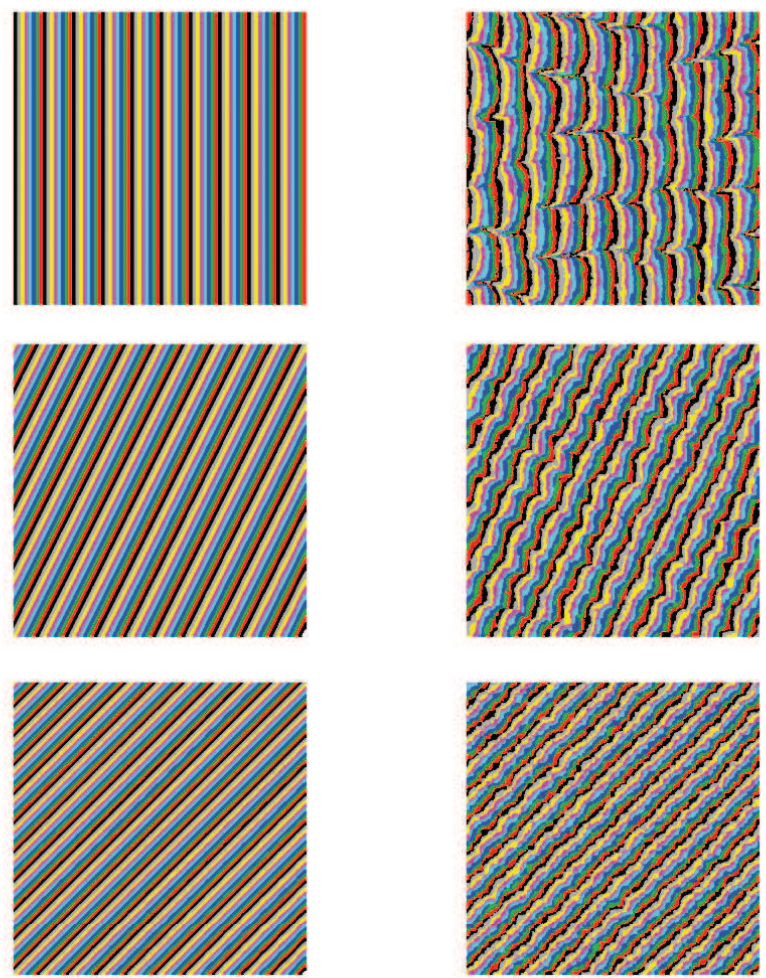

Figure 5: Snapshots of surface morphology in simple-cubic lattice simulations. Each row shows snapshots at 0 $\mathrm{ML}$ and a $80 \mathrm{ML}$. The deposition rate $F=10^{-3}$, the bond strength $e_{b}=11$ and the rejection rate $v=0.9$. The top pair corresponds to the case of perfectly flat steps with periodic and helical boundary conditions imposed. The next two pairs correspond to cases where steps are oriented at 26.6 and 45 degrees. Plots in the second columns correspond to data points in Fig. 6. 


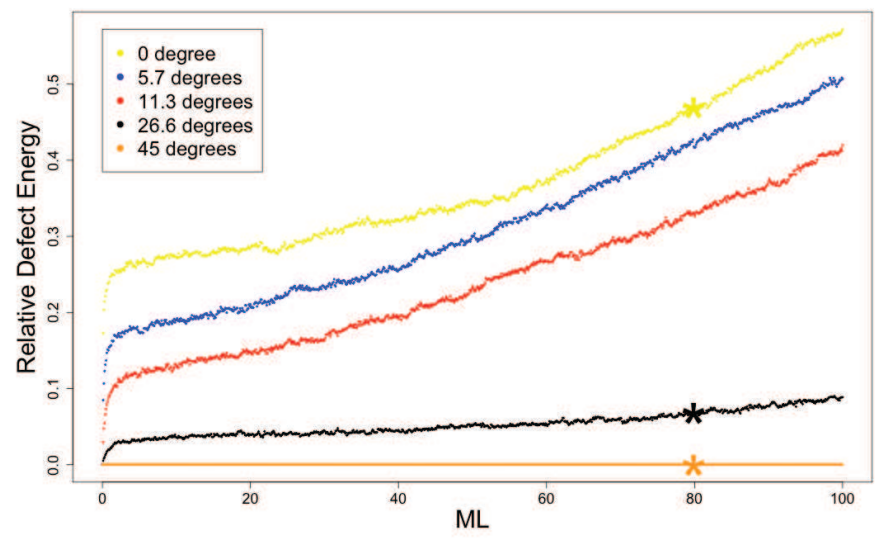

Figure 6: Evolution of the relative defect energy in simple-cubic lattice simulations. The top curve corresponds to a no-kink condition, where the angle of tilt is zero. The bottom curve corresponds to the case with the most kinks, where the angle of tilt is 45 degrees. As we increase the angle of tilt, we observe a stabilizing effect.

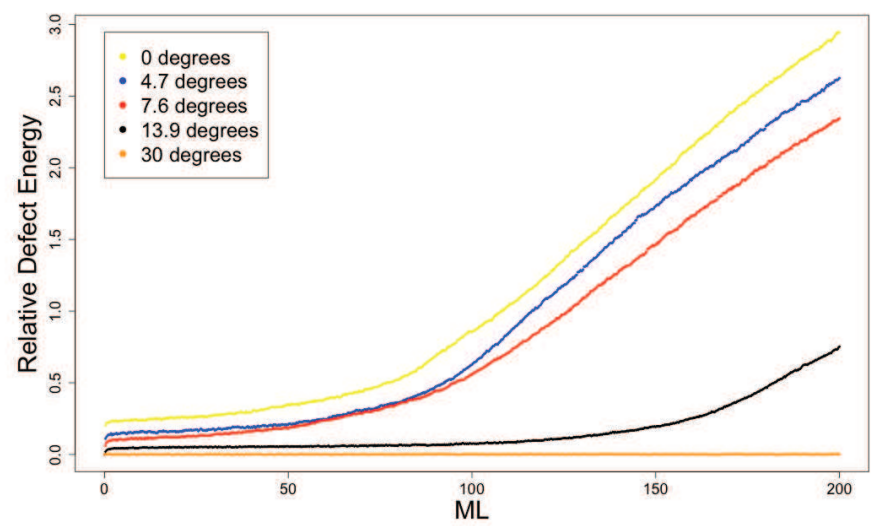

Figure 7: Evolution of the relative defect energy in triangular lattice simulations. The top curve corresponds to a no-kink condition, where the angle of tilt is zero. The bottom curve corresponds to the case with the most kinks, where the angle of tilt is 30 degrees. As we increase the angle of tilt, we observe a stabilizing effect.

where $\bar{\gamma}_{0}$ is the non-reconstructed surface energy of the mean edge orientation, $Z$ is the lattice coordination and $N_{i}$ is the total number of atoms with $i$ bonds, as a function of monolayers grown for the various orientations. Thus, the defect energy also serves as a good measure of the degree to which the interface has formed meanders. We repeat this for the triangular lattice in Fig. 7, where we again see that it is the orientation favored for equilibrium configurations that is most susceptible to the meandering instability.

Our final result is to repeat the calculations for the hexagonal lattice. The equilibrium shape for this lattice features hexagonal structures with zigzag facets, consistent with the Wulff shape shown in Fig. 2. However, Figs. 8 and 9 show that it is the armchair orientation that is most prone to the meandering instability. This is consistent with the proposition that the perturbations to the interface have a surface energy governed by the 

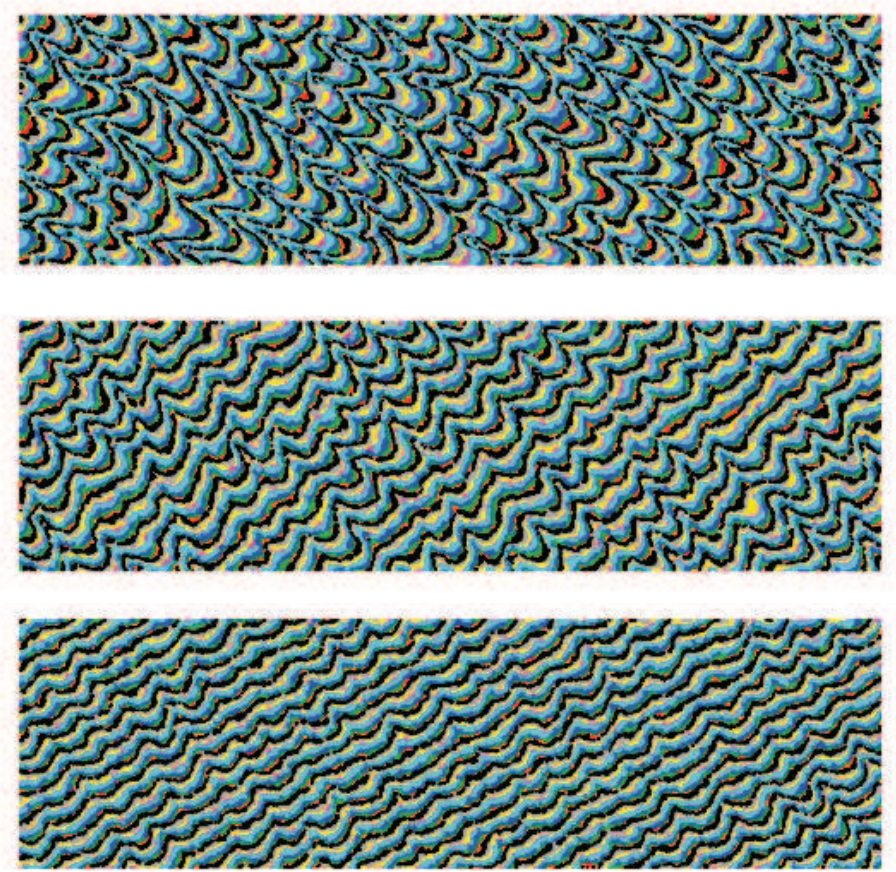

Figure 8: Contour plots of surface height after $100 \mathrm{ML}$ of growth on a 900 by 300 section of hexagonal lattice with 180 steps. The deposition rate $F=10^{-7}$, the bond strength $e_{b}=10$ and the rejection rate $v=.96$. The top figure had the steps constrained to the armchair orientation, while the bottom figure was constrained to the zigzag orientation, with the third image at an intermediate orientation. These correspond to the data points shown in Fig. 9.

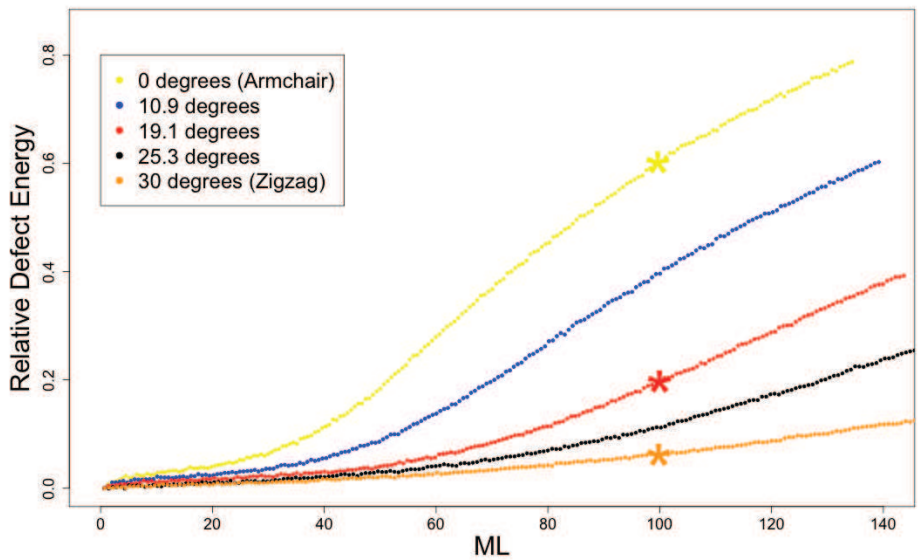

Figure 9: Surface energy as a function of time for the hexagonal version of the Bales-Zangwill instability. The top curve corresponds to the armchair orientation, indicating that this is the most unstable orientation, while zigzag corresponds to the lowest curve, indicating that this is the most stable orientation.

non-reconstructed edge energy (2.5) in Fig. 2. 


\section{Conclusion}

The results in the previous section are consistent with the non-reconstructed defect energy being the dominant factor governing the evolution of disturbances to the moving fronts in the presence of a step-edge barrier. There may, however, be other factors that explain or contribute to the explanation for the observed behavior. For example, in addition to the anisotropic surface energy, it may be the case that the asymmetry in attachment kinetics is influenced by the anisotropy. If so, it seems likely that this effect too could be traced back to the singular behavior of the broken bond density as a function of orientation.

Further work examining the impact of these observations on the non-reconstructed evolution of crystals would be of interest. Simulations using molecular dynamics, KMC and/or phase-field crystal would seem well suited to this purpose.

\section{Acknowledgments}

We wish to acknowledge support from NSF-DMS-1613729. We would also like to thanks Ken Elder and Steve Wise for many helpful discussions regarding this work.

\section{References}

[1] J. S. Langer, "Models of Pattern Formation in First-Order Phase Transitions", Dir. Cond. Matt. Phys. 1 165-86 (1986).

[2] W. J. Boettinger, J. A. Warren, C. Beckermann, and A. Karma, "Phase-Field Simulation of Solidification", Annu. Rev. of Mater. Res. 32, 163-94 (2002).

[3] N. Provatas and K. Elder, Phase-Field Methods in Materials Science and Engineering, Wiley (2001).

[4] N. Moelans, B. Blanpain, and P. Wollants, "An introduction to phase-field modeling of microstructure evolution", Calphad 32, 268-94 (2008).

[5] G. S. Bales and A. Zangwill, "Morphological instability of a terrace edge during step-flow growth", Phys. Rev. B 41, 5500 (1990).

[6] G. Ehrlich and F. G. Hudda, "Atomic View of Surface Self-Diffusion: Tungsten on Tungsten", J. Chem. Phys. 44, 1039-1049 (1966).

[7] R. L. Schwoebel and E. J. Shipsey, "Step Motion on Crystal Surfaces", J. App. Phys. 37, 36823686 (1966).

[8] N. Ashcroft and N. D. Mermin. Solid state physics, Holt, Rinehart and Winston, (1976).

[9] J. R. Hook and H. E. Hall,Solid-State Physics, Wiley (2006)

[10] J.K. Mackenzie, A.J.W. Moore, and J.F. Nicholas, "Bonds Broken at Atomically Flat Crystal Surfaces I: Face-Centered and Body-Centered Cubic Crystals", J. Phys. Chem. Solids 23, 185196 (1962).

[11] C. Herring, "Some Theorems on the Free Energies of Crystal Surfaces", Phys. Rev. 82 87-93 (1951).

[12] C. K. Gan and D. Srolovitz, "Trends in graphene edge properties and flake shapes: a firstprinciples study", Phys. Rev. B 81125445 (2010). 
[13] Y. Liu, A. Dobrinsky, and B. Yakobson, "Graphene Edge from Armchair to Zigzag: The Origins of Nanotube Chirality?", Phys. Rev. Lett. 105235502 (2010).

[14] V. Artyukhov, Y. Liu, and B. Yakobson, "Equilibrium at the edge and atomistic mechanisms of graphene growth", Proceedings of the National Academy of Sciences 109 15136-15140 (2012).

[15] W. K. Burton, N. Cabrera, and F. C. Frank "The Growth of Crystals and the Equilibrium Structure of their Surfaces", Phil. Trans. R. Soc. Lond. A 243 299-358 (1951).

[16] V. Blum, R. Gehrke, F. Hanke, P. Havu, V. Havu, X. Ren, K. Reuter, and M. Scheffler, Comput. Phys. Commun. 180, 2175 (2009).

[17] J. P. Perdew, K. Burke, and M. Ernzerhof, Phys. Rev. Lett. 77, 3865 (1996).

[18] A. Tkatchenko and M. Scheffler, Phys. Rev. Lett. 102, 073005 (2009).

[19] K. Suenaga and M. Koshino, "Atom-by-atom spectroscopy at graphene edge", Nature 468, 1088-1090 (2010).

[20] H. Weyl, "Ueber die Gleichverteilung von Zahlen mod.", Math. Ann. 77, 313-352 (1916).

[21] K.J. Laidler and M.C. King, "The development of Transition-State Theory," J. Phys. Chem 87 2657-2664 (1983).

[22] G. Henkelman and H. Jonsson, "Long time scale kinetic Monte Carlo simulations without lattice approximation and predefined event table," J. Chem. Phys. 115 9657-9666 (2001).

[23] E. Ising, "Beitrag zur Theorie des Ferromagnetismus", Z. Phys. 31253258 (1925).

[24] R. J. Baxter, Exactly solved models in statistical mechanics, London: Academic Press Inc. (1982).

[25] T. P. Schulze, “Efficient Monte Carlo Simulation", J. Comp. Phys. 227 2455-2462 (2008)

[26] F. Nita and A. Pimpinelli, "When Smoothening Makes It Rough: Unhindered Step-Edge Diffusion and the Meandering Instability on Metal Surfaces", Phys. Rev. Lett. 95106104 (2005) 